\title{
Rabbit common calcanean tendon as an animal model: ultrasonographic anatomy and morphometry
}

\author{
A. Skalec ${ }^{1}$, M. Janeczek' ${ }^{1}$ I. Janus² ${ }^{2}$ A. Chrószcz¹ , R. Henklewski \\ ${ }^{1}$ Department of Animal Physiology and Biostructure, Wroclaw University of Environmental and Life Sciences, \\ Faculty of Veterinary Medicine, Wroclaw, Poland \\ ${ }^{2}$ Department of Pathology, Wroclaw University of Environmental and Life Sciences, Faculty of Veterinary Medicine, \\ Wroclaw, Poland \\ ${ }^{3}$ Department of Surgery, Wroclaw University of Environmental and Life Sciences, Faculty of Veterinary Medicine, \\ Wroclaw, Poland
}

[Received: 30 April 2015; Accepted: 25 May 2015]

Background: The aim of the present study was to evaluate usefulness of ultrasonography in assessment of rabbit common calcanean tendon, to describe its ultrasonographic anatomy and to perform morphometric analysis of this structure. Materials and methods: Fifteen skeletally-matured New Zealand rabbits were used in the study. Ultrasonographic examinations of common calcanean tendon (CCT) were performed in longitudinal and transverse planes from caudal approach. Sagittal diameters of superficial digital flexor tendon and CCT were measured on longitudinal scans. Sagittal and transverse diameter, cross-sectional area and perimeter of the CCT were assessed on transverse scans. Statistical analysis was performed using StatisticaPL software (StatSoft ${ }^{\circledR}$, Poland).

Results: In longitudinal images structure of CCT was clearly visualised. Its superficial hypoechoic part corresponds to superficial digital flexor tendon and deeper hyperechoic to gastrocnemius tendon. In transverse images cross-sectional area presented varied echotexture. Proximally, CCT was rounded in transverse section and became slightly wider and flattered distally. Statistical analysis showed no differences between results obtained from right and left hindlimb ( $p>0.05)$. Measurements of sagittal diameter of CCT obtained in transverse planes were significantly higher than sagittal diameter measurements obtained in longitudinal plane in corresponding locations $(p<0.001)$. All performed measurements showed a growing trend with the increasing distance from the calcaneal tuber. Conclusions: Ultrasonography is suitable technique for assessment of CCT in a rabbit model and provides satisfactory images for morphometrical evaluation. (Folia Morphol 2016; 75, 1: 93-100)

Key words: anatomy, morphometry, ultrasound examination

\section{INTRODUCTION}

Tendinopathies, in the sense of the broad spectrum of pathological conditions of tendons, are the most frequently occurring musculoskeletal disorders among human and equine athletes in variety of sport disciplines, although they also affect individuals with low physical activity [13] and pleasure riding horses. It was stated, that in humans, commonly affected 
are Achilles, patellar, rotator cuff and medial/lateral elbow tendons [19]. In flat horse races and National Hunt racehorses, tendons and ligaments injuries account for $46 \%$ of all musculoskeletal injuries [32]. Majority of those conditions occur in frontlimbs, most commonly affecting superficial digital flexor tendon $[1,14]$. Tendon injury was also found to be the most common reason for retirement in racing Thoroughbreds [18] leading to significant losses in horse-racing industry. Due to prolonged rehabilitation period and high re-injury rate in equine $[7,24]$ and human patients [13], tendon pathologies continue to challenge clinicians and veterinary surgeons. An in-depth knowledge of aetiology, risk factors and molecular, cellular and tissue mechanisms involved in tendon injuries is essential for estimating new treatment modalities. For those reasons experimental studies on animal models play a crucial role in tendinopathy research. A valid animal model needs to consistently replicate the clinical, histopathological and functional characteristics of tendon disorders [19] along with being accessible to assess with imaging techniques commonly used in human and equine medicine for eventual comparison. Considering that the tissue specimens of injured tendons are not available for histopathological examination in clinical practice, the ultrasound and magnetic resonance imaging are the gold standard in diagnosing tendon disorders, with ultrasonography being cost-effective, non-invasive and generally accessible $[10,11,21]$. Several animal models are currently used in tendinopathy research including laboratory animals, like rabbits $[5,23,25,26,29,33]$, rats $[9,15,17]$ and mice [8] as well as companion animals, like horses $[2,3,31]$ and dogs [20]. Despite the fact that veterinary patients are increasingly recognised as translational models of human tendinopathies $[19,30]$ their use in experimental protocols seems controversial and is not accepted in several countries due to ethical issues. Therefore laboratory animal models are indispensable for tendinopathy research with rabbits common calcanean tendon (CCT) being frequently used, due to its superficial localisation, relatively large size and practical as well as economic reasons [12]. Superficial localisation makes rabbit CCT easily accessible for ultrasound examination.

Accurate interpretation of sonographic images requires a profound anatomical knowledge of the examined area to avoid misinterpretation or overinterpretation. There are some differences in the cru- ral musculature between humans and quadripedal mammals which influence the anatomy of calcanean tendon. The most significant is the absence of superficial digital flexor in humans.

In a rabbit, CCT consists of tendons of medial and lateral heads of the gastrocnemius muscle, with negligible contribution from the soleus muscle (those components correspond to human calcanean tendon), the superficial digital flexor tendon and the accessory calcaneal ligament from the semitendinosus muscle (a thin tendinous band joined with medial gastrocnemius tendon). In a region of musculotendinous junctions the medial gastrocnemius tendon is located caudally, the lateral gastrocnemius tendon craniolaterally and the superficial digital flexor tendon craniomedially. Coursing distally they rotate, so that the superficial digital flexor tendon runs side by side with the medial gastrocnemius and then becomes localised caudally to the others. Fibres of both gastrocnemius tendons converge at about $93 \%$ of overall course to their insertion on the calcaneal tuber [6]. The superficial digital flexor tendon extends over the calcaneal tuber separated from it by subtendinous calcaneal bursa of flexor digitorum superficialis.

Although rabbit CCT was assessed ultrasonographically in a course of several tendinopathy studies, there is a lack of detailed literature description of its normal ultrasound images that may be used for reference. The aim of the present study was to evaluate usefulness of ultrasonography in assessment of rabbit $\mathrm{CCT}$, to describe its ultrasonographic anatomy and to perform morphometric analysis of this structure.

\section{MATERIALS AND METHODS}

The protocol of this study was approved by II Local Ethics Committee of Wroclaw University of Environmental and Life Sciences (decision no. 107/2014). Fifteen skeletally-matured female New Zealand rabbits of approximately $12-15$ months age and $3.45-4.10 \mathrm{~kg}$ body weight were used in the experiment. The CCTs of both hindlimbs in each rabbit were examined. Animals were anaesthetised by intramuscular injection of $0.4 \mathrm{mg} / \mathrm{kg}$ medetomidine (Cepetor ${ }^{\circledR}$, ScanVet Poland). The skin over both CCTs was shaved, moistened with surgical spirit and covered with ultrasound coupling gel to prevent the presence of air between the transducer and skin surface, what could influence the quality of obtained images. Animals were positioned in lateral recumbence with the examined hindlimb flexed in the tarsal join at approximately 


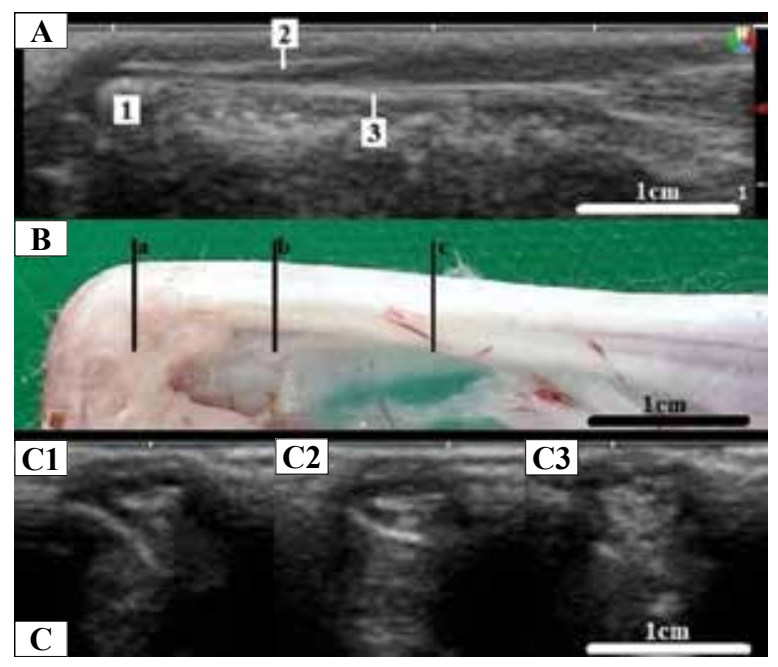

Figure 1. A. Longitudinal ultrasonographic image of rabbit common calcanean tendon (CCT). Proximal is to the right, caudal is to the top. 1 - calcaneal tuber, 2 - superficial digital flexor tendon, 3 - gastrocnemius tendon; B. CCT at postmortem dissection* in a position corresponding to longitudinal ultrasonographic view. $\mathrm{a}, \mathrm{b}, \mathrm{c}$ - lines indicate the locations of ultrasound probe for obtaining transverse scans; C. Transverse ultrasonographic images of CCT; $\mathrm{C} 1$ - above calcaneal tuber (corresponding to line a), C2 $-1 \mathrm{~cm}$ proximally (line b), C3 $-2 \mathrm{~cm}$ proximally (line c); *Dissection was performed on a rabbit euthanized in a course of a different experiment, not included in this study.

$90^{\circ}$ to assure adequate tension of the tendon during examination. After ultrasonographic assessment all rabbits recovered from anaesthesia within oxygen chamber equipped with a heating mat (Kruuse ${ }^{\circledR}$ ). Postanaesthesia protocol included oral application of nutrition supplement (Rodicare Instant ${ }^{\circledR}$, Alfavet).

Ultrasonographic images were captured using a Sonoscape $\mathrm{S} 6 \mathrm{~V}^{\circledR}$ unit with linear-array transducer with frequency range $5-13 \mathrm{MHz}$. All images were obtained by 1 operator to avoid interoperator variables during image acquisition. Tendons were scanned in longitudinal and transverse planes from caudal approach. In longitudinal plane the ultrasound probe was placed slightly to the medial side to assure good visualisation of the superficial digital flexor tendon. The orientation point in longitudinal projection was the calcaneal tuber. Tendons were scanned in 3 transverse projections in 1-cm intervals starting directly at calcaneal insertion and proceeding $2 \mathrm{~cm}$ proximally (Fig. 1). As there was no orientation point on transverse scans, care was taken to precisely place the ultrasound probe in desired locations, determined by a manual calliper.

Analysis of all obtained images was undertaken by the same operator who performed image acqui-

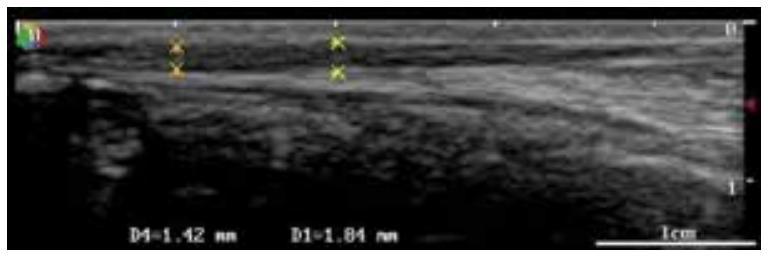

Figure 2. Longitudinal ultrasonographic image of rabbit common calcanean tendon. Proximal is to the right, caudal is to the top. Sagittal diameter measurements of superficial digital flexor tendon $1 \mathrm{~cm}$ (D4) and $2 \mathrm{~cm}$ (D1) proximally from calcaneal tuber.

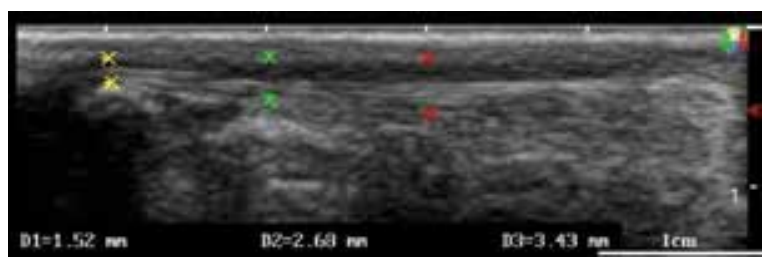

Figure 3. Longitudinal ultrasonographic image of rabbit common calcanean tendon. Proximal is to the right, caudal is to the top. Sagittal diameter measurements of common calcanean tendon above (D1), $1 \mathrm{~cm}$ (D2) and $2 \mathrm{~cm}$ (D3) proximally from calcanean tuber.

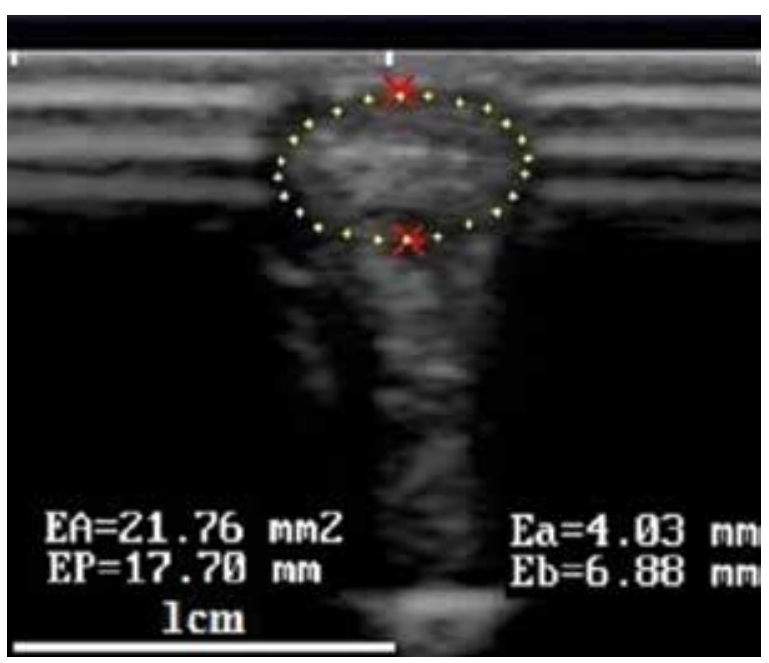

Figure 4. Transverse ultrasonographic image of common calcanean tendon $1 \mathrm{~cm}$ proximally from calcanean tuber. Caudal is to the top; $\mathrm{EA}$ - cross-sectional area; EP — perimeter; Ea — sagittal diameter; $\mathrm{Eb}$ - transverse diameter.

sition. In longitudinal images the caudocranial dimension (sagittal diameter) of the superficial digital flexor tendon was measured in 2 locations: $1 \mathrm{~cm}$ and $2 \mathrm{~cm}$ proximally from the calcaneal tuber (Fig. 2). The combined caudocranial dimension (sagittal diameter) of the CCT was measured directly above calcaneal tuber, $1 \mathrm{~cm}$ and $2 \mathrm{~cm}$ proximally (Fig. 3). In transverse images the caudocranial (sagittal) and lateromedial dimension (transverse diameter) of the CCT was measured along with cross-sectional area and perimeter (Fig. 4). Image analysis was performed on stored scans the day after obtaining images. All 
measurements were obtained 3 times for calculating the mean value used for statistical analysis.

\section{Statistical analysis}

The statistical analysis was performed using StatisticaPL software (StatSoft ${ }^{\circledR}$, Poland). The following test were used: Shapiro-Wilk test to determine the normality of the data distribution, Student's t test to determine differences between results obtained from each hindlimb and differences between measurements obtained from longitudinal and transverse images, ANOVA test to determine differences between the measurements at various distance from the calcaneal tuber, Pearson's test to assess the correlation between the variables. The level of statistical significance was set at $p \leq 0.05$.

\section{RESULTS}

In longitudinal images structure of the CCT was clearly visualised. Due to echogenicity it can be divided into superficial hypoechoic part and deeper hyperechoic component. Superficial structure corresponds to the superficial digital flexor tendon and the deeper one, with fine, parallel hyperechoic collagen fibres, to the gastrocnemius tendon. The musculotendinous junction of gastrocnemius component was well defined but transition of the superficial digital flexor muscle to tendon was not visible in those images. The distinction between tendons of medial and lateral head of gastrocnemius was impossible to obtain in this plane. In transverse images it was impossible to distinguish different tendinous components of the CCT and cross-sectional area presented varied echotexture due to anisotropy caused by 3 tendon units with fibres running in slightly different directions. Proximally, the CCT was rounded in transverse section and became slightly wider and flattered distally. The soleus muscle and the accessory ligament of semitendinosus muscle were not detected ultrasonographically. Summarised measurements obtained in every scanning position are presented in Table 1.

The statistical analysis showed no differences between results obtained from right and left hindlimb ( $p>0.05$ ). Measurements of sagittal diameter of the CCT obtained in transverse planes were significantly higher than sagittal diameter measurements obtained in longitudinal plane in corresponding locations ( $p<0.001$, Fig. 5). A positive correlation was shown between sagittal diameter of CCT measured on transverse and longitudinal scans above the calcaneal tu- ber ( $p<0.05, r=0.39$ ) and $1 \mathrm{~cm}$ from the calcaneal tuber ( $p<0.05, r=0.41$ ), no correlation was found $2 \mathrm{~cm}$ from the calcaneal tuber $(p>0.05)$. In the longitudinal plane a positive correlation was found between measurements taken $1 \mathrm{~cm}$ and $2 \mathrm{~cm}$ proximally from the calcaneal tuber for both sagittal diameter of the superficial digital flexor tendon $(p<0.05$, $r=0.57)$ and sagittal diameter of the CCT ( $p<0.05$, $r=0.57)$ but there was no positive correlation with measurements taken directly above calcaneal tuber ( $p>0.05$ ). In the transverse plane a positive correlation was found between measurements taken above the calcaneal tuber and $1 \mathrm{~cm}$ from the tuber $(p<0.05$, $r=0.43$ for the sagittal diameter, $p<0.05, r=0.64$ for the transverse diameter of the CCT). All measurements showed a growing trend with the increasing distance from the calcaneal tuber (Fig. 6). Except the measurement of the transverse diameter in the transverse planes above and $1 \mathrm{~cm}$ from the calcaneal tuber, all measurements showed significant differences ( $p<0.001)$.

\section{DISCUSSION}

Rabbits CCT often serves as an animal model for the assessment of new treatment modalities in tendinopathy research. As musculoskeletal ultrasonography is currently routinely used for diagnosing and evaluation of tendon disorders, the constant effort is made to obtain better standardisation of scanning methods to decrease operator dependence [22]. To the best of our knowledge, currently there is no published paper describing the complete methodology of ultrasonographic examination of rabbits CCT and for this reason, we had to establish our own examination protocol. It is well known that adequate standardisation of transducer positioning is essential for obtaining comparable images [11]. To avoid influence of variables such as placing the ultrasound probe, selection of the image for scan acquisition and selection of the tendons boundaries for measurements, in our experiment the same operator was performing ultrasound examination and image analysis. In the study investigating the repeatability of diagnostic ultrasonography in the assessment of equine superficial digital flexor tendons, authors demonstrate that no significant interoperator difference in image acquisition was identified, although there were significant differences during image analysis [27]. They conclude that one operator should undertake image analysis, but different operators may undertake image acquisition. 
Table 1. Examined tendons with ultrasound plane; localisation of measurement; mean, maximum, minimum values and standard deviations (SD)

\begin{tabular}{|c|c|c|c|c|c|c|}
\hline Anatomical structure & $\begin{array}{l}\text { Ultrasound } \\
\text { plane }\end{array}$ & $\begin{array}{l}\text { Exact } \\
\text { location }\end{array}$ & $\begin{array}{l}\text { Mean value } \\
{[\mathrm{mm}]}\end{array}$ & $\begin{array}{c}\text { Minimum } \\
\text { value }[\mathrm{mm}]\end{array}$ & $\begin{array}{c}\text { Maximum } \\
\text { value }[\mathrm{mm}]\end{array}$ & $\begin{array}{l}\text { SD } \\
{[\mathrm{mm}]}\end{array}$ \\
\hline CCT — sagittal diameter & Longitudinal & Above calcaneal tuber & 1.11 & 0.75 & 1.52 & 0.11 \\
\hline ССТ — sagittal diameter & Longitudinal & $\begin{array}{l}1 \mathrm{~cm} \text { proximally from } \\
\text { calcaneal tuber }\end{array}$ & 2.81 & 1.92 & 3.68 & 0.37 \\
\hline SDFT — sagittal diameter & Longitudinal & $\begin{array}{l}1 \mathrm{~cm} \text { proximally from } \\
\text { calcaneal tuber }\end{array}$ & 1.34 & 0.92 & 1.86 & 0.20 \\
\hline СCT — sagittal diameter & Longitudinal & $\begin{array}{l}2 \mathrm{~cm} \text { proximally from } \\
\text { calcaneal tuber }\end{array}$ & 3.41 & 2.34 & 4.27 & 0.38 \\
\hline SDFT — sagittal diameter & Longitudinal & $\begin{array}{l}2 \mathrm{~cm} \text { proximally from } \\
\text { calcaneal tuber }\end{array}$ & 1.57 & 1.00 & 2.18 & 0.22 \\
\hline CCT — transverse diameter & Transverse & $\begin{array}{l}\text { Directly at calcaneal } \\
\text { insertion }\end{array}$ & 5.96 & 4.20 & 8.89 & 0.95 \\
\hline CCT — sagittal diameter & Transverse & $\begin{array}{l}\text { Directly at calcaneal } \\
\text { insertion }\end{array}$ & 3.35 & 2.35 & 5.02 & 0.46 \\
\hline CCT - perimeter & Transverse & $\begin{array}{l}\text { Directly at calcaneal } \\
\text { insertion }\end{array}$ & 15.2 & 11.00 & 21.11 & 2.19 \\
\hline CCT — cross sectional area $\left[\mathrm{mm}^{2}\right]$ & Transverse & $\begin{array}{l}\text { Directly at calcaneal } \\
\text { insertion }\end{array}$ & 15.89 & 8.04 & 28.4 & 4.08 \\
\hline ССТ — transverse diameter & Transverse & $\begin{array}{l}1 \mathrm{~cm} \text { proximally from } \\
\text { calcaneal tuber }\end{array}$ & 6.22 & 4.69 & 8.66 & 0.81 \\
\hline ССТ — sagittal diameter & Transverse & $\begin{array}{l}1 \mathrm{~cm} \text { proximally from } \\
\text { calcaneal tuber }\end{array}$ & 3.81 & 2.51 & 5.19 & 0.59 \\
\hline CCT — perimeter & Transverse & $\begin{array}{l}1 \mathrm{~cm} \text { proximally from } \\
\text { calcaneal tuber }\end{array}$ & 16.14 & 3.57 & 21.18 & 2.21 \\
\hline СCT — cross sectional area $\left[\mathrm{mm}^{2}\right]$ & Transverse & $\begin{array}{l}1 \mathrm{~cm} \text { proximally from } \\
\text { calcaneal tuber }\end{array}$ & 18.72 & 10.13 & 30.71 & 4.41 \\
\hline ССТ — transverse diameter & Transverse & $\begin{array}{l}2 \mathrm{~cm} \text { proximally from } \\
\text { calcaneal tuber }\end{array}$ & 7.91 & 5.94 & 10.89 & 1.16 \\
\hline CCT — sagittal diameter & Transverse & $\begin{array}{l}2 \mathrm{~cm} \text { proximally from } \\
\text { calcaneal tuber }\end{array}$ & 5.34 & 2.92 & 8.88 & 1.26 \\
\hline CCT — perimeter & Transverse & $\begin{array}{l}2 \mathrm{~cm} \text { proximally from } \\
\text { calcaneal tuber }\end{array}$ & 21.3 & 14.70 & 30.37 & 3.36 \\
\hline CCT — cross sectional area $\left[\mathrm{mm}^{2}\right]$ & Transverse & $\begin{array}{l}2 \mathrm{~cm} \text { proximally from } \\
\text { calcaneal tuber }\end{array}$ & 33.86 & 13.62 & 72.52 & 12.10 \\
\hline
\end{tabular}

CCT — common calcanean tendon; SDFT — superficial digital flexor tendon

In rabbit CCT animal model investigators usually use either the superficial digital flexor tendon [23, $25,26]$ or the gastrocnemius tendon [33]. In the study of comparative anatomy of rabbit and human Achilles tendons with magnetic resonance and ultrasound imaging, the authors concluded that transverse and longitudinal ultrasonographic images of the gastrocnemius-soleus complex in a rabbit, although obtained, were difficult to interpret due to extensive anisotropy and small dimension [6]. As components of the CCT rotate along their long axis on the course to calcaneal tuber, preserving the perpendicular angle between each tendon and ultrasound beam is impossible. This may lead to artificial decrease in tendons echogenicity [10,11, 21, 22] and inconclusive images in transverse plane. It stands in agreement with our results - in transverse images single components (gastrocnemius tendons, superficial digital flexor tendon) of CCT could not be precisely identified. On longitudinal images the distinction between lateral and medial gastrocnemius tendons was impossible, although in our opinion the superficial digital flexor tendon could be distinguished from the gastrocnemius complex. In a study of shock wave effect on 

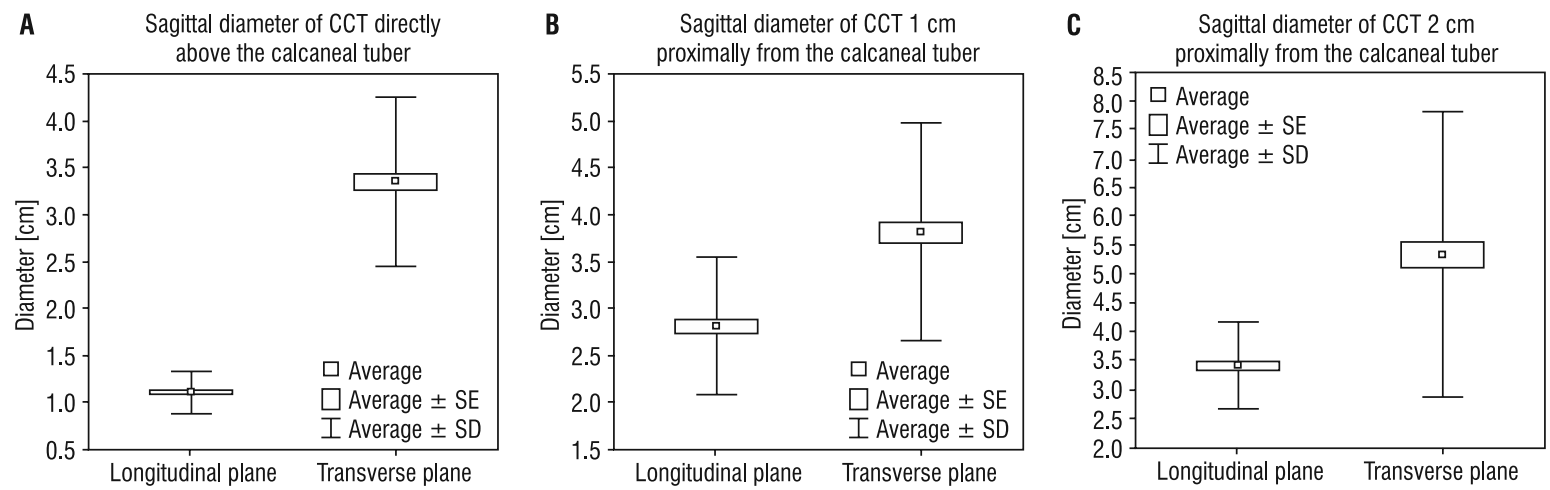

Figure 5. Comparison of sagittal diameter measurements of common calcanean tendon (CCT) in the longitudinal plane and transverse plane in corresponding locations; SE — standard error; SD — standard deviation; SDFT — superficial digital flexor tendon.
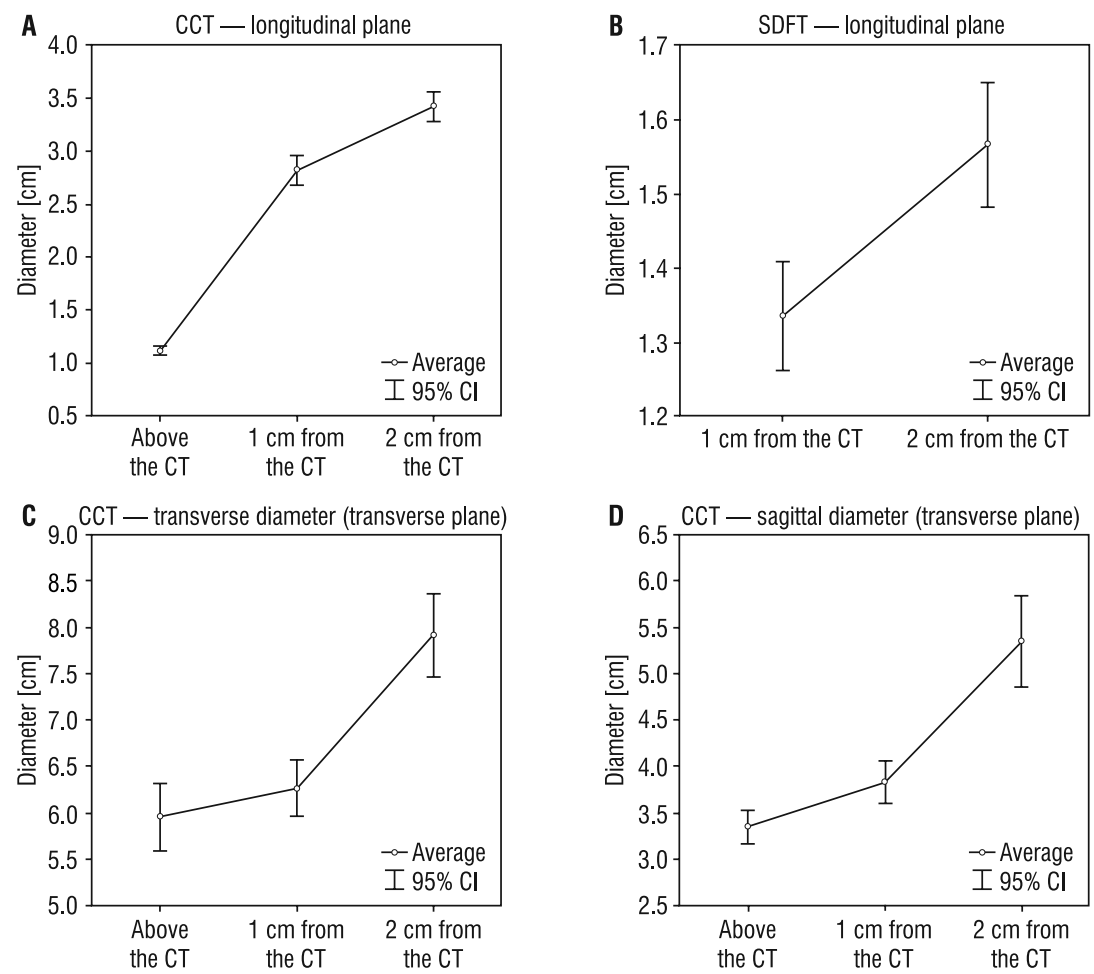

Figure 6. The sagittal (A, B, D) and transverse (C) diameters of common calcanean tendon (CCT) and superficial digital flexor tendon (SDFT) in longitudinal (A, B) and transverse (C, D) planes in different locations; $\mathrm{Cl}$ — confidence intervals; $\mathrm{CT}$ — calcaneal tuber.

rabbit Achilles tendon, authors were able to assess Achilles tendon diameter and echostructure [28]. Another research groups investigating the superficial digital flexor tendon with surgically created lesion used ultrasonography in assessment of tendon healing response $[23,25,26]$. Those investigators agree that transverse images and cross-sectional area of the superficial digital flexor tendon was not diagnostic. But on longitudinal sections echogenicity and homogeneity along with tendon diameter could be assessed [26]. Moreover, despite small tendon dimension they report differences in diameter between treated and control tendons that could be measured and were statistically relevant [23]. It is in agreement with our results that the superficial digital flexor tendon can be assessed in longitudinal plane and in transverse planes it is only possible to examine the CCT as a whole.

According to the results of our study, we conclude that the contralateral CCTs of the same rabbit can serve as an ultrasonographic control to each other. It also means that right and left tendons can be chosen randomly for treatment and control groups in this ani- 
mal model. In the paper describing reproducibility of sonographic measurement of thickness and echogenicity of the plantar fascia authors demonstrate, that fascial sagittal diameter measured in longitudinal and transverse scans are highly comparable [4]. This is in contrast with our results; sagittal diameter of the CCT measured in transverse plane is significantly higher than in longitudinal plane in the corresponding locations. Those differences can be caused by better visualisation of subcutaneous tissue in longitudinal images, due to larger contact area with the transducer on the longitudinal scans and by the varied echotexture of the tendon on transverse scans, which make it more difficult to distinguish with subcutaneous tissue. The lack of positive correlation between sagittal diameter of the tendon in transverse and longitudinal planes $2 \mathrm{~cm}$ proximal to the calcaneal tuber, along with the lack of positive correlation between measurements obtained on transverse scans in this location and those obtained in two other locations, suggest that this area of the CCT may varies significantly between individuals. This is in contrast to measurements of the sagittal diameter of the CCT over the calcaneal tuber that seems constant irrespective of tendon size measured in more proximal locations. It should be reminded, that measurements undertaken on ultrasound scans not necessarily correspond to actual dimensions. Those measurements can only be used to assess changes in repetitive examinations in the course of the experiment and for estimating standard reference values. Our results led us to the conclusion that if study protocol includes creation of tendon injury and ultrasound evaluation of tendon healing response, longitudinal images seem more accurate for exact localisation of lesion and assessment of fibre pattern and echogenicity. Transverse images in the region of lesion can serve as a source of additional information.

A limiting factor of the present study is the frequency range of ultrasound probe. Currently, there are commercially available linear transducers of working frequencies reaching $18-22 \mathrm{MHz}$, which allow obtaining high resolution images of superficial structures. However, as the traditional orthopaedic linear probes are commonly accessible and used in tendon research we decided to choose it for our study.

Despite this limitation, the present study indicate, that ultrasonography is suitable technique for assessment of CCT in a rabbit model and provide satisfactory images for morphometrical evaluation.

\section{CONCLUSIONS}

Ultrasonography is suitable technique for assessment of CCT in a rabbit model and provides satisfactory images for morphometrical evaluation.

\section{ACKNOWLEDGEMENTS}

The research was supported by statutory research and development activity funds assigned to Faculty of Veterinary Medicine, Wroclaw University of Environmental and Life Sciences.

Publication supported by Wroclaw Centre of Biotechnology, programme the Leading National Research Centre (KNOW) for years 2014-2018.

\section{REFERENCES}

1. Avella CS, Ely ER, Verheyen KLP, Price JS, Wood JLN, Smith RKW (2009) Ultrasonographic assessment of the superficial digital flexor tendons of National Hunt racehorses in training over two racing seasons. Equine Vet J, 41: 449-454. doi: 10.1100/2012/472023.

2. Carvalho AM, Badial PR, Alvarez LEC, Yamada ALM, Borges AS, Deffune E, Hussni CA, Alves ALG (2013) Equine tendonitis therapy using mesenchymal stem cells and platelet concentrates: a randomized controlled trial. Stem Cell Res Ther, 4: 85. doi: 10.1186/scrt236.

3. Carvalho AM, Yamada ALM, Golim MA, Alvarez LEC, Hussni CA, Alves ALG (2013) Evaluation of mesenchymal stem cell migration after equine tendonitis therapy. Equine Vet J, 46: 635-638. doi: 10.1111/evj.12173.

4. Cheng J-W, Tsai W-C, Yu T-Y, Huang K-Y (2012) Reproducibility of sonographic measurement of thickness end echogenicity of the plantar fascia. J Clin Ultrasound, 40: 14-19. doi: 10.1002/jcu.20903.

5. Derby BM, Reichensperger J, Chambers C, Bueno RA, Suchy H, Neumeister MW (2012) Early growth response factor-1: expression in a rabbit flexor tendon scar model. Plast Reconstr Surg, 129: 435e-442e. doi: 10.1097/ /PRS.0b013e3182402d81.

6. Doherty GP, Koike Y, Uhthoff HK, Lecompte M, Trudel G (2006) Comparative anatomy of rabbit and human Achilles tendons with magnetic resonance and ultrasound imaging. Comp Med, 56: 68-74.

7. Dyson SJ (2004) Medical management of superficial digital flexor tendonitis: a comparative study in 219 horses (1992-2000). Equine Vet J, 36: 415-419. doi: $10.2746 / 0425164044868422$.

8. Freedman BR, Sarver JJ, Buckley MR, Voleti PB, Soslowsky $\sqcup$ (2014) Biomechanical and structural response of healing Achilles tendon to fatigue loading following acute injury. J Biomech, 47: 2028-2034. doi: 10.1016/j. jbiomech.2015.02.058.

9. Gereli A, Akgun U, Uslu S, Agir I, Ates F, Nalbantoglu U (2014) The effect of organic silicon injection on Achilles tendon healing in rats. Acta Orthop Traumatol Turc, 48: 346-354. doi: 10.3944/AOTT.2014.3162.

10. Grassi W, Cervini C (1998) Ultrasonography in rheumatology: an evolving technique. Ann Rheum Dis, 57: 268-271. doi: 10.1136/ard.57.5.268.

11. Grassi W, Filippucci E, Farina A, Cervini C (2000) Sonographic imaging of tendons. Arthritis Rheum, 43: 969-976. 
doi: 10.1002/1529-0131(200005)43:5<969::AID-ANR2 > 3.0.CO;2-4.

12. Hast MW, Zuskov A, Soslowsky $\sqcup$ (2014) The role of animal models in tendon research. Bone Joint Res, 3: 193-202. doi: 10.1302/2046-3758.36.2000281.

13. Kader D, Saxena A, Movin T, Maffulli N (2002) Achilles tendinopathy: some aspects of basic sciences and clinical management. Br J Sports Med, 36: 239-249. doi: 10.1136/ bjsm.36.4.239.

14. Kasashima Y, Takahashi T., Smith RKW, Goodship AE, Kuwano A, Ueno T, Hirano S (2004) Prevalence of superficial digital flexor tendonitis and suspensory desmitis in Japanese Thoroughbred flat racehorses in 1999. Equine Vet J, 36: 346-350. doi: 10.2746/0425164044890580.

15. Kaux J-F, Janssen L, Drion $P$, Nusgens $B$, Libertiaux V, Pascon $F$, Heyeres A, Hoffmann A, Lambert C, Le Goff C, Denoel V, Defraigne J-O, Rickert M, Crielaard J-M, Colige A (2014) Vascular endothelial growth factor-111 (VEGF-111) and tendon healing: preliminary results in a rat model of tendon injury. Muscles Ligaments Tendons J, 4: 24-28.

16. Kidd JA, Lu KG, Frazer ML (2014) Atlas of equine ultrasonography. Wiley Blackwell, Chichester.

17. Killian ML, Cavinatto L, Shah SA, Sato EJ, Ward SR, Havlioglu N, Galatz LM, Thomopoulos S (2014) The effects of chronic unloading and gap formation on tendon-to-bone healing in a rat model of massive rotator cuff tears. J Orthop Res, 32: 439-447. doi: 10.1002/jor.22519.

18. Lam KH, Parkin TDH, Riggs CM, Morgan KL (2007) Descriptive analysis of retirement of Thoroughbred racehorses due to tendon injuries at Hong Kong Jockey Club (1992-2004). Equine Vet J, 39: 143-148. doi: 10.2746/042516407X159132.

19. Lui PPY, Maffulli N, Rolf C, Smith RKW (2011) What are the validated animal models for tendinopathy? Scand J Med Sci Sports, 21: 3-17. doi: 10.1111/j.1600-0838.2010.01164.x.

20. Manning CN, Schwartz AG, Liu W, Xie J, Havlioglu N, Sakiyama-Elbert SE, Silva MJ, Xia Y, Gelberman RH, Thomopoulos S (2013) Controlled delivery of mesenchymal stem cells and growth factors using a nanofiber scaffold for tendon repair. Acta Biomat, 9: 6905-6914. doi: 10.1016/j. actbio.2013.02.008.

21. Martinoli C, Bianchi S, Dahmane M, Pugliese F, Bianchi-Zamorani MP, Valle M (2002) Ultrasound of tendons and nerves. Eur Radiol, 12: 44-55. doi:10.1007/s00330001-1161-9.

22. Micu MC, Serra S, Fodor D, Crespo M, Naredo E (2011) Inter-observer reliability of ultrasound detection of tendon abnormalities at the wrist and ankle in patients with rheumatoid arthritis. Rheumatology, 50: 1120-1124. doi:10.1093/rheumatology/keq441.
23. Moshiri A, Oryan A (2011) Structural and functional modulation of early healing of full-thickness superficial digital flexor tendon rupture in rabbits by repeated subcutaneous administration of exogenous human recombinant basic fibroblast growth factor. J Foot Ankle Surg, 50: 654-662. doi: 10.1053/j.jfas.2011.05.002.

24. O'Meara B, Bladon B, Parkin TDH, Fraser B, Lischer CJ (2010) An investigation of relationship between race performance and superficial digital flexor tendonitis in the Thoroughbred racehorse. Equine Vet J, 42: 322-326. doi: 10.1111/j.0425-1640.2009.00021.x.

25. Oryan A, Moshiri A, Parizi AHM, Jahromi AR (2012) Repeated administration of exogenous sodium-hyaluronate improved tendon healing in an in vivo transection model. J Tissue Viability, 21: 88-102. doi: 10.1016/j. jtv.2012.06.002.

26. Oryan A, Moshiri A (2011) A long term study on the role of exogenous human recombinant basic fibroblast growth factor on the superficial digital flexor tendon healing in rabbits. J Musculoskelet Neuronal Interact, 11: 185-195.

27. Pickersgill CH, Marr CM, Reid SWJ (2001) Repeatability of diagnostic ultrasonography in the assessment of equine superficial digital flexor tendon. Equine Vet J, 33: 33-37. doi: 10.2746/042516401776767494.

28. Rompe JD, Kirkpatrick CJ, Kullmer K, Schwitalle M, Krischek O (1998) Dose-related effects of shock waves on rabbit tendo achillis. J Bone Joint Surg, 80B: 546-552.

29. Sekouris N, Kefalas A, Soultanis K, Diamantopoulou K, Karagiannopoulou G, Soucacos P, Zoubos A (2013) Cellular response of the tendon sheath in tendon injury: experimental research in New Zealand Rabbits. J Trauma Treat, S4: 004. doi: 10.4172/2167-1222.S4-004.

30. Volk SW, Theoret C (2013) Translating stem cell therapies: The role of companion animals in regenerative medicine. Wound Repair Regen, 21: 382-394. doi: 10.1111/ wrr. 12044

31. Watts AE, Nixon AJ, Yeager AE, Mohammed HO (2012) A collagenase gel/physical defect model for controlled induction of superficial digital flexor tendonitis. Equine Vet J, 44: 576-586. doi: 10.1111/j.2042-3306.2011. 00471.x.

32. Williams RB, Harkins LS, Hammond CJ, Wood JLN (2001) Racehorse injuries, clinical problems and fatalities recorded on British racecourses from flat racing and National Hunt racing during 1996, 1997 and 1998. Equine Vet J, 33: 478-486. doi: 10.2746/04251640177625480.

33. Young RG, Butler DL, Weber W, Caplan Al, Gordon SL, Fink DJ (1998) Use of mesenchymal stem cells in a collagen matrix for Achilles tendon repair. J Orthop Res, 16: 406-413. 